Revista Ambientale

Revista da Universidade Estadual de Alagoas/UNEAL e-ISSN 2318-454X, Ano 13, Vol. 13 (1), 2021

\title{
Aspectos biológicos e produtivos do tomateiro (Solanum lycopersicum) sob adubação húmica
}

\section{Biological and productive aspects of tomato (Solanum lycopersicum) under humic fertilization}

\author{
Maria Lúcia Barbosa ${ }^{(1)}$; Rubens Pessoa de $\operatorname{Barros}^{(2)}$; Rodrigo Almeida Pinheiro ${ }^{(3)}$. \\ ${ }^{(1)}$ ORCID: 0000-0002-8721-2588; Graduanda em Ciências biológicas; Universidade Estadual de Alagoas- \\ UNEAL; E-mail: luciia.barbosa@live.com. \\ (2)ORCID: 0000-0003-0140-1570; Professor do Departamento de Ciências Biológicas da Universidade Estadual \\ de Alagoas-UNEAL/Campus I; E-mail: pessoa.rubens@gmail.com. \\ ${ }^{(3)}$ ORCID: 0000-0001-5642-5065; Mestrando em Produção Vegetal; Universidade Federal dos Vales do \\ Jequitinhonha e Mucuri-UFVJM; E-mail: rodrigo6450@ gmail.com.
}

E-mail do autor correspondente: luciia.barbosa@ live.com

Resumo - A espécie Solanum lycopersicum L., é uma frutífera pertencente ao gênero Solanum que integra a família Solanaceae, sendo uma das olerícolas de destaque econômico e com ampla distribuição geográfica, presente em diferentes habitats e diversas condições ambientais, com grande aceitabilidade e valor nutricional em muitas dietas, diante desses fatos, muitos estudos foram direcionados para a produção. Este estudo objetivou avaliar os aspectos biológicos e produtivos S. lycopersicum variedade Santa Clara em diferentes doses de humus de minhoca. O estudo foi desenvolvido no Campus I da Universidade Estadual de Alagoas, em Arapiraca$\mathrm{AL}$, em casa de vegetação com $50 \%$ em retenção de luminosidade, durante os meses de novembro de 2019 a janeiro de 2020 . No estudo foram avaliadas diferentes dosagens de húmus de minhoca. $\mathrm{O}$ arranjo experimental utilizado foi do tipo inteiramente casualizado (DIC), com quatro tratamentos e cinco repetições, sendo eles: $\mathrm{T} 1-$ solo $+100 \mathrm{~g}$ de humus; $\mathrm{T} 2-$ solo $+75 \mathrm{~g}$ de humus; T3- solo + 50g de humus; T4-. solo/testemunha. As variáveis foram submetidas à Análise de Variância, e para comparação de médias utilizou-se o teste de Tukey $(\mathrm{P}<0,05)$, pelo Software Sisvar. As diferentes doses de húmus de minhoca não apresentaram diferenças significativas no desenvolvimento e produção de tomate $S$. lycopersicum, apenas o tratamento solo (testemunha) apresentou resultados inferiores às diferentes doses de húmus de minhoca. São necessários outros estudos para verificar a eficiência do substrato em S. lycopersicum, assim como sua combinação com outros substratos e adubos.

Palavras-chave: Compostos orgânicos. Florescimento. Frutos. Produtividade. Solanáceas.

Abstract - The species Solanum lycopersicum L., is a fruit planta belonging to the Solanum genus that integrates the Solanaceae family, being one of the economically prominent vegetables and with wide geographical distribution, present in different habitats and different environmental conditions, with great acceptability and nutritional value many diets, in view of these facts, many studies were directed towards production. This study aimed to evaluate the biological and productive aspects of S. lycopersicum variety Santa Clara in different doses of earthworm humus. The study was developed at Campus I of the State University of Alagoas, in 


\section{Revista Ambientale \\ Revista da Universidade Estadual de Alagoas/UNEAL \\ e-ISSN 2318-454X, Ano 13, Vol. 13 (1), 2021}

Arapiraca - AL, in a greenhouse with $50 \%$ retention of luminosity, during the months of November 2019 to January 2020. The experimental arrangement used was entirely randomized (DIC), with four treatments and five repetitions, being: T1 - soil $+100 \mathrm{~g}$ of humus; T2- soil + $75 \mathrm{~g}$ of humus; T3- soil $+50 \mathrm{~g}$ of humus; $\mathrm{T} 4-$. solo / witness. The variables were subjected to Analysis of variation, and Tukey's test $(\mathrm{P}<0.05)$ was used to compare means, using the Sisvar Software. The different doses of earthworm humus did not differentiate differences in the development and production of S. lycopersicum, only the soil treatment (control) presented lower results than the different doses of earthworm humus. Further studiesshould out to verify the efficiency of the substrate in S. lycopersicum, as well as its combination with other substrates and fertilizers.

Keywords: Organic compounds, Flowering, Fruits, productivity, Solanaceae.

\section{Introdução}

As frutas são condimentos importantes para a complementação da alimentação do brasileira, além de estarem associadas ao desenvolvimento e crescimento da indústria (FERREIRA, 2004).

O tomate (Solanum lycopersicum L.), é uma frutífera pertencente ao gênero Solanum que integra a família Solanaceae, sendo uma das olerícolas de destaque econômico e com ampla distribuição geográfica, presente em diferentes habitats e diversas condições ambientais, com grande aceitabilidade e valor nutricional em muitas dietas (CARVALHO; PAGLIUCA, 2007; ALVARENGA, 2013).

O desenvolvimento e a produtividade das plantas são processos complexos que dependem da interação de fatores ambientais, fisiológicos e genéticos (Aghaie et al., 2018). E neste sentido, S. lycopersicum é tido como uma cultura de alto custo de produção e exigente (SILVA et al., 2003).

De acordo com Costa et al. (2018), S. lycopersicum é a segunda hortaliça mais cultivada e consumida no mundo e apresenta diversos tipos de frutos, sendo os mais encontrados no mercado os vermelhos e graúdos.

A planta de $S$. lycopersicum origina um fruto muito apreciado mundialmente, caracterizado por ser carnoso e suculento. Além disso, a cultura de S. lycopersicum também possui grande importância nutricional em virtude dos nutrientes de sua composição, sendo que entre os principais está o carotenoide licopeno responsável pela sua coloração (FILGUEIRA, 2007).

A classificação de $S$. lycopersicum pode ser feita utilizando-se critérios como formato, grau de maturação, tamanho e presença de defeitos, sendo estes graves ou leves, havendo diferentes níveis de tolerância para cada um deles conforme o produto a ser produzido (SENAR, 2015). S. lycopersicum fornece vitamina C, pró-vitamina A e antioxidantes, essenciais ao ser humano (REIFSCHNEIDER et al., 2014).

Outra importante característica nutricional dos frutos de $S$. lycopersicum é sua elevada concentração de licopeno, considerado um importante carotenoide de ação antioxidante que protege o organismo de radicais livres responsáveis por desencadear o surgimento do câncer de próstata e de outras doenças crônicas (PALOMO et al., 2010; MOLTIVA et al., 2014). 


\section{Revista Ambientale \\ Revista da Universidade Estadual de Alagoas/UNEAL e-ISSN 2318-454X, Ano 13, Vol. 13 (1), 2021}

Uma diversidade de resíduos de origem orgânica é gerada pelas atividades agropecuárias, estes resíduos podem causar impactos ao meio ambiente se não forem manejados de forma correta. Neste sentido a adubação feita de maneira correta torna-se uma alternativa mais segura e acessível para produtores e consumidores (SANTOS et al., 2012). A adubação orgânica é uma prática que não deve ser dispensada no cultivo de $S$. lycopersicum origina um fruto mais saudável e suculento. Além disso, favorecendo seu estado nutricional, melhorando sua aparência e forma (FILGUEIRA, 2007).

Os benefícios da aplicação de matéria orgânica são atribuídos à liberação gradativa de nutrientes e à estruturação do solo, favorecendo o crescimento do sistema radicular de $S$. lycopersicum (INCAPER, 2010).

Adubos orgânicos como o húmus de minhoca é um material em decomposição consumido pelas minhocas que, no seu trato digestivo, potencializa as propriedades químicas e físicas do solo, aumentando a disponibilidade dos elementos para as plantas, e acelera o processo de compostagem. (RIBEIRO, 2001).

As substâncias húmicas possuem ação benéfica no metabolismo da planta e nas características do solo. Ainda, como aumenta a capacidade de reter água em 20 vezes a sua massa, a decomposição da matéria fornecendo nutrientes para o crescimento da planta. Além de possuir fatores benéficos que necessitam de estudos para esclarecimentos. (CANELLAS; ZANDONADI, 2006).

O Brasil ocupa a oitava posição na classificação mundial de produção de $S$. lycopersicum, com um volume de aproximadamente de 3,7 milhões de toneladas, com produtividade de 63,7 t/ha-1, devido isso, deve-se desenvolver cada vez mais alternativas de manejo para aumento da produção de $S$. lycopersicum buscando alternativas limpas e que preservem os ecossistemas (IBGE, 2015).

Partindo da premissa do desenvolvimento biológico de plantas tomates em resposta a adubações, o estudo teve como objetivo avaliar os aspectos biológicos e produtivos Solanum lycopersicum variedade Santa Clara em diferentes doses de húmus de minhoca.

\section{Procedimento metodológico}

O estudo foi desenvolvido no Campus I da Universidade Estadual de Alagoas em Arapiraca- AL, em casa de vegetação com $50 \%$ em retenção de luminosidade, durante os meses de novembro de 2019 a janeiro de 2020 . Com as seguintes coordenadas geográficas de latitude: 0945'09' S; e de longitude: 36³9'40' W, com altitude de $264 \mathrm{~m}$.

O município de Arapiraca está situado na região agreste do Estado de Alagoas, que apresenta condições edafoclimáticas com temperaturas de $22^{\circ} \mathrm{C}$ (mínima) e de $30^{\circ} \mathrm{C}$ (máxima), com precipitação média anual de $665 \mathrm{~mm}$, e com a radiação ultravioleta máxima de índice UV 9 (SEMA-RHDMET, 2020). O clima da região é do tipo As', determinando como clima tropical e quente, segundo a classificação de Köppen e Geiger de 2006.

O preparo das mudas foi realizado utilizando-se 20 vasos de polietileno com capacidade de $5 \mathrm{~L}$ e área $25 \times 25 \mathrm{~cm}$, com diferentes doses de húmus de minhoca, e foram dispostas cinco sementes por vaso, totalizando 100 sementes. As sementes foram adquiridas comercialmente da ISLA ${ }^{\circledR}$. Após 25 dias da emergência (DAE) foi realizado o desbaste dos vasos, permanecendo uma planta por vaso. 
No estudo foram avaliadas diferentes dosagens de húmus de minhoca. $\mathrm{O}$ arranjo experimental utilizado foi inteiramente casualizado (DIC), com quatro tratamentos e cinco repetições, sendo eles: $\mathrm{T}_{1}-$ solo $+100 \mathrm{~g}$ de humus; $\mathrm{T}_{2}-$ solo $+75 \mathrm{~g}$ de humus; $\mathrm{T}_{3}-$ solo $+50 \mathrm{~g}$ de humus; $\mathrm{T}_{4}-$. solo/testemunha.

As variáveis do desenvolvimento biológico e monitoradas foram diâmetro do caule (DC), número folhas (NFL), altura da planta, número de botões floras (NBF) e número de frutos (NF).

Os dados foram submetidos à análise de Variância (ANOVA), e para comparação de médias utilizou-se o teste de Tukey $(\mathrm{P}<0,05)$, pelo Software SISVAR versão 5.6 (FERREIRA, 2011).

\section{Resultados e discussão}

De acordo com a análise de variância, o Teste F em cada variável analisada demonstrou significância para os tratamentos das dosagens de húmus nas variáveis de altura da planta (AP), número de folhas (NFL), diâmetro do caule (DC) e o número de botões florais (NBF) produzidos pelas plantas de $S$. lycopersicum. Em relação ao número de frutos (NFTS), o teste apontou um coeficiente de variação negativo em relação as outras variáveis observadas. Tal fato deve ser levado em consideração quando comparado o número de botões florais produzidos e o número de frutos vingados e contados após a colheita (Tabela 1).

Tabela 1. Análise de variância (ANOVA) com as respectivas fontes de variação das dosagens de húmus de minhoca em S. lycopersicum (Solanaceae).

\begin{tabular}{cccccc}
\hline FV & GL & SQ & QM & F & $\mathbf{C V}_{(\%)}$ \\
\hline AP (cm) & 3 & 593,20 & 197,73 & 3,73 & 7,02 \\
NFL (u) & 3 & 46,75 & 15,58 & 2,60 & 13,19 \\
DC (cm) & 3 & 0,018 & 0,006 & 1,43 & 10,66 \\
NBF (u) & 3 & 171,37 & 57,12 & 10,39 & 7,04 \\
NFTS (u) & 3 & 575,75 & 191,91 & 9,32 & 37,64
\end{tabular}

Legenda: $(\mathrm{cm})$ : centímetros; (u): unidade; (FV): fontes de variação; (GL): graus de liberdade; (SQ): soma dos quadrados; (QM): quadrado médio; $(\mathrm{F})$ : teste F; $(\mathrm{CV}(\%)$ : coeficiente de variação. Altura da planta (AP); número folhas (NFL); diâmetro do caule (DC); número de botões floras (NBF) e número de frutos (NFTS).

O teste de Tukey a 5\% demonstrou que estatisticamente as variáveis de número de número de folhas (NFL), diâmetro do caule (DC) e número de botões florais não se diferenciaram, ou seja, as diferentes dosagens de húmus de $100 \mathrm{~g}, 75 \mathrm{~g}$ e $50 \mathrm{~g}$ não se diferenciaram da testemunha (Tabela 2).

Dentre os tratamentos utilizados, o mais influente no crescimento e altura das plantas (AP) de $S$. lycopersicum foi a testemunha, e os aproximados sendo os tratamentos de $50 \mathrm{~g} \mathrm{e}$ $75 \mathrm{~g}$. Em relação a variável do número de frutos (NFTS) as dosagens de húmus não apresentaram diferenças entre si, mas a testemunha apresentou uma média abaixo das doses de húmus utilizadas, como o esperado (Tabela 2). 
Revista Ambientale

Revista da Universidade Estadual de Alagoas/UNEAL

e-ISSN 2318-454X, Ano 13, Vol. 13 (1), 2021

Tabela 2. Médias do teste de Tukey a 5\% no experimento de S. lycopersicum (Solanaceae) para as dosagens de húmus de minhoca.

\begin{tabular}{cccccc}
\hline TRATAMENTOS & AP $(\mathbf{c m})$ & NFL $(\mathbf{c m})$ & DC (u) & NBF (u) & NF (u) \\
\hline $\mathbf{1 0 0} \mathbf{g}$ & $95,24 \mathrm{~b}$ & $16,91 \mathrm{a}$ & $0,59 \mathrm{a}$ & $28,93 \mathrm{a}$ & $14,20 \mathrm{a}$ \\
$\mathbf{7 5} \mathbf{~ g}$ & $103,80 \mathrm{~b} \mathrm{a}$ & $17,22 \mathrm{a}$ & $0,57 \mathrm{a}$ & $32,39 \mathrm{a}$ & $18,00 \mathrm{a}$ \\
$\mathbf{5 0} \mathbf{g}$ & $105,10 \mathrm{~b} \mathrm{a}$ & $19,57 \mathrm{a}$ & $0,65 \mathrm{a}$ & $35,91 \mathrm{a}$ & $12,60 \mathrm{a}$ \\
TESTEMUNHA & $110,42 \mathrm{a}$ & $20,51 \mathrm{a}$ & $0,61 \mathrm{a}$ & $36,05 \mathrm{a}$ & $3,40 \mathrm{~b}$
\end{tabular}

Nas colunas, médias seguidas pelas mesmas letras não diferem estatisticamente entre si, pelo teste de Tukey $(\mathrm{P}<0,05)$. Legenda: altura da planta (AP); número folhas (NFL); diâmetro do caule (DC); número de botões floras (NBF) e número de frutos (NFTS).

Em experimento com diferentes tipos de adubação orgânica para Ocimum basilicum, sendo um deles, o húmus de minhoca misturado ao solo, da Silva et al. (2020) observaram que não houve diferença entre os tratamentos com cama de aviário principalmente, esterco bovino e esterco caprino nas variáveis de crescimento e produção de massa fresca de seca das plantas de $O$. basilicum, sendo um resultado semelhante deste estudo com $S$. lycopersicum, onde as diferentes doses de humus de minhoca não influenciaram no crescimento e produção de $S$. lycopersicum.

Em experimento realizado por Linhares et al. (2014), avaliaram o crescimento Vigna unguiculata sob diferentes dosagens de húmus de minhoca, que proporcionou o aumento do número de flores e diâmetro do caule. As doses de húmus para $S$. lycopersicum não se diferenciaram para as variáveis de diâmetro do caule (DC) e número de botões floras (NBF), sendo positivos de acordo com os testes aplicados.

Da Silva Araújo et al. (2020) utilizaram húmus de minhoca para a germinação de Eruca sativa, sendo o tratamento utilizando somente o húmus influenciou positivamente na germinação de E. sativa. Porém, o substrato combinado com esterco caprino e bovino afetou negativamente a germinação de E. sativa não sendo recomendado a combinação destes substratos.

Da Silva Araújo et al. (2018) observaram em seus tratamentos utilizados em experimento com E. sativa, sendo eles solo + esterco bovino e solo + húmus de minhoca, não houve uma distinção considerável entre os tratamentos. Porém, o tratamento de solo + húmus de minhoca influenciaram positivamente em variáveis como altura da planta, mas em relação a produção de massa fresca e seca, outros adubos conseguiram proporcionar melhor desempenho para E. sativa.

\section{Considerações finais}

As diferentes doses de húmus de minhoca não influenciaram nos aspectos biológicos e produtivos de S. lycopersicum, apenas o tratamento utilizando apenas solo (testemunha) apresentou resultados abaixo das médias das diferentes doses de húmus de minhoca. 
São necessários outros estudos para verificar a eficiência do húmus de minhoca como substrato para S. lycopersicum, assim como sua combinação com outros substratos/adubos.

\section{Agradecimentos}

A Deus, pelo discernimento para desenvolver esta pesquisa. Aos meus pais, parentes e amigos que me apoiaram nesta trajetória.

Aos membros do grupo de estudos ambientais e etnobiológicos - GEMBIO, Rodrigo Almeida Pinheiro, Daniel Rocha Santos, João Pedro Silva Oliveira e Lívia Gibrinde pelo apoio e dedicação quando não pude estar presente para os devidos cuidados com meu experimento.

Ao Prof. Dr. Rubens Pessoa de Barros pela orientação e apoio.

\section{Conflito de interesses}

Os autores deste manuscrito não declararam conflitos de interesse. 


\section{Referências}

AGHAIE, P.; TAFRESHI, A. A. H.; EBRAHIMI, M. A.; HAERINADAB, M. Tolerance evaluation and clustering of fourteen tomato cultivars grown under mild and severe drought conditions. Scientia Horticulturae, Amsterdam, v. 232, n. 32, p. 1-12, 2018.

ALVARENGA, M. A. R. Tomate: produção em campo, em casa de vegetação e em hidroponia. 2. ed. Lavras: UFLA, 2013. 455 p.

CANELLAS, L. P.; ZANDONADI, D. B.; OLIVARES, F. L.; FAÇANHA, A. R. Efeitos fisiológicos de substancias húmicas: o estímulo às H+ - ATPases. Viçosa: Fernandes, 2006.

CARVALHO, J. L.; PAGLIUCA, L. G. Tomate: Um mercado que não para de crescer globalmente. Revista Hortifruti Brasil, Piracicaba-SP, v. 58, n. 1, p. 6-14, 2007.

COSTA, E. S. P.; SANTOS, C. A.; ROCHA, M. C.; CARMO, M. G. Caracterização física, físico-química e morfoagronômica de acessos de tomate cereja sob cultivo orgânico. Revista de Ciências Agrárias, Belém, v. 61, p. 1-8, 2018.

DA SILVA ARAÚJO, A.; DA SILVA, D. J.; DE SOUZA SILVA, A. V.; DA SILVA, A. T.; DE LIRA, A. C. B.; DE BARROS, R. P. Potencial germinativo de sementes de rúcula (Eruca sativa L., Brassicaceae) em diferentes substratos. Diversitas Journal, v. 5, n. 3, p. 1495$1503,2020$.

DA SILVA ARAÚJO, A.; DE SOUZA SILVA, A. V.; DA SILVA, A. T.; DA SILVA, D. J. Efeitos da adubação orgânica e mineral no cultivo da rúcula (Eruca sativa Miller., Brassicaceae). Anais do VIII Encontro Científico Cultural - ENCCULT 2018 Universidade Estadual de Alagoas Campus II - Santana do Ipanema/AL. P 151 - 154.

DA SILVA, J. E.; PINEHIRO, R. A.; BARBOSA, J. P. F.; DOS SANTOS CABRAL, M. J.; SANTOS, D. R.; DE BARROS, R. P. Eficiência de substratos orgânicos no desenvolvimento do Manjericão (Ocimum basilicum L.). Diversitas Journal, v. 5, n. 3, p. 1442-1450, 2020.

DOS SANTOS, J. O.; DE SOUSA SANTOS, R. M.; BORGES, M. D. G. B.; FERREIRA, R. T. F. V.; SALGADO, A. B.; DOS SANTOS SEGUNDO, O. A. A evolução da agricultura orgânica. Revista Brasileira de Gestão Ambiental, Pombal, PB, v. 6, n. 1, p. 35-41, 2012.

FERREIRA, D. F. Sisvar: a computer statistical analysis system. Ciência e agrotecnologia, v. 35, n. 6, p. 1039-1042, 2011.

FERREIRA, R.M.S. Características de Qualidade do Tomate de mesa (Lycopersicon esculentum Mill.) Cultivado nos Sistemas Convencional e Orgânico Comercializado na Região Metropolitana de Curitiba. Tese (Pós Graduação em Tecnologia de Alimentos) Universidade Federal do Paraná, Brasil, 2004. 
Revista Ambientale

Revista da Universidade Estadual de Alagoas/UNEAL

e-ISSN 2318-454X, Ano 13, Vol. 13 (1), 2021

FILGUEIRA, F. A. R. Novo Manual de Olericultura: agrotecnologia moderna na produção e comercialização de hortaliças. 3. ed: Viçosa: UFV, 2007, p. 412.

IBGE. Instituto Brasileiro de Geografia e Estatística. Levantamento sistemático da produção agrícola, Rio de Janeiro, v. 29, n. 8, p. 1-79, ago. 2015.

INCAPER. Instituto Capixaba de Pesquisa, Assistência Técnica e Extensão Rural. Tomate. Vitória, ES, 2010.

LINHARES, P. P. A.; SILVA, J. N.; SOUZA, J. A.; SOUZA, T. P.; ANDRADE, R.; MEDEIROS, A. C.; MARACAJÁ, P. B. Crescimento do feijão-caupi sob adubação orgânica em condições edafoclimáticas de Catolé do Rocha-PB. Revista GVAA, Paraíba, v. 8, n. 1, 2014

MOLTIVA, M. J.; MACIÀ, A.; ROMERO, M. P.; LABRADOR, A.; DOMÍNGUEZ, A.; PEIRÓ, L. Optimisation and validation of analytical methods for the simultaneous extraction of antioxidants: Application to the analysis of tomato sauces. Food Chemistry, London, v. 163, p. 234-243, 2014.

PALOMO, I.; MOORE-CARRASCO, R.; CARRASCO, G.; VILLALOBOS, P.; GUZMÁN, L. Tomato consumption prevents the development of cardiovascular events and cancer: epidemiologic antecedents and action mechanisms. Idesia, Arica, v. 28, p. 121-129, 2010.

REIFSCHNEIDER, F.J.B.; NASS, L.L.; HEINRICH, G.A.; CLÁUDIA S.C.; RIBEIRO, C.S.C.; HENZ, P.G.; EUCLIDES FILHO, K.; BOITEUX, L.S.; RITSCHEL, P.; FERRAZ, R.M.; QUECINI, V. Uma pitada de biodiversidade na mesa dos brasileiros. Brasília:1 ed., 156p., 2014.

RIBEIRO, G. T.; PAIVA, H. D.; JACOVINE, L. A. G.; TRINDADE, C. Produção de mudas de Eucalipto. Viçosa: Aprenda fácil. 2001.

SENAR-AR/SP (Serviço Nacional de Aprendizagem Rural). Processamento do tomate. Cartilha, p. 36. 2015.

SILVA, J. B. C.; GIORDANO, L. B.; FUROMOT, O.; BOITEUX, L. S.; FRANÇA, F. H.; BÔAS G. L. V.; BRANCO, M. C.; MEDEIROS, M. A.; MAROUELLI, W.; SILVA, W. L. C.; LOPES, C. A.; ÁVILA, A. C.; NASCIMENTO, W. M.; PEREIRAI, W. Tomate industrial: Brasilia, DF: Embrapa Hortaliças, 2003. (Sistema de Produção, 3). 